\title{
Numerical Computation of a Generalized Exponential Integral Function*
}

\author{
By W. F. Breig and A. L. Crosbie
}

\begin{abstract}
Series expansions and recurrence relations suitable for numerical computation are developed for the generalized exponential integral functions. Tables of these functions are presented in the microfiche section of this issue.
\end{abstract}

1. Introduction. A generalization of the exponential integral function can be defined by

$$
\begin{aligned}
& \mathcal{E}_{1}(\tau, \beta)=\int_{1}^{\infty}\left(t^{2}+\beta^{2}\right)^{-1 / 2} \exp \left[-\tau\left(t^{2}+\beta^{2}\right)^{1 / 2}\right] d t, \\
& \mathcal{E}_{2}(\tau, \beta)=\int_{1}^{\infty} t^{-2} \exp \left[-\tau\left(t^{2}+\beta^{2}\right)^{1 / 2}\right] d t, \\
& \mathcal{E}_{3}(\tau, \beta)=\tau \int_{1}^{\infty} \mathcal{E}_{2}(\tau t, \beta / t) d t .
\end{aligned}
$$

These functions arise in the study of radiative transfer in a two-dimensional planar medium ([1] [4]). For example, the function $\varepsilon_{1}(\tau, \beta)$ is the kernel of the Fredholm integral equations describing isotropic scattering $([1]-[3])$ and radiative equilibrium [4]. The functions, $\varepsilon_{1}(\tau, \beta), \varepsilon_{2}(\tau, \beta)$ and $\varepsilon_{3}(\tau, \beta)$, are two-dimensional analogs of the exponential integral function

$$
E_{n}(\tau)=\int_{1}^{\infty} t^{-n} \exp (-\tau t) d t
$$

$n=1,2,3$. Since the two functions are identical when $\beta=0, \varepsilon_{n}(\tau, \beta)$ may be considered a generalized exponential integral.

2. Recurrence Relations. As with the one-dimensional exponential integrals, recursive formulas are useful in the numerical evaluation of $\varepsilon_{n}(\tau, \beta)$. A simple integration by parts of (1a) yields

$$
\varepsilon_{2}(\tau, \beta)=\exp \left[-\tau\left(1+\beta^{2}\right)^{1 / 2}\right]-\tau \mathcal{E}_{1}(\tau, \beta)
$$

where $\varepsilon_{2}(0, \beta)=1$. Equation (3) is analogous to the recursion formula for the exponential integral function

$$
E_{2}(\tau)=\exp (-\tau)-\tau E_{1}(\tau)
$$

Received February 13, 1973.

AMS (MOS) subject classifications (1970). Primary 33A10, 65O20, $65 \mathrm{~A} 05$.

Key words and phrases. Exponential integral, special functions.

* This research was supported by grant GK-35859 from the National Science Foundation.

Copyright $\odot$ 1974, American Mathematical Society 
The function $\varepsilon_{3}(\tau, \beta)$ can be expressed in terms of $\varepsilon_{2}(\tau, \beta)$ by the insertion of (3) into (1c). Thus

$$
\begin{aligned}
\mathcal{E}_{3}(\tau, \beta)= & \tau \int_{1}^{\infty} \exp \left[-\tau\left(t^{2}+\beta^{2}\right)^{1 / 2}\right] d t \\
& -\tau^{2} \int_{1}^{\infty} \int_{1}^{\infty} t^{2}\left(t^{2} x^{2}+\beta^{2}\right)^{-1 / 2} \exp \left[-\tau\left(t^{2} x^{2}+\beta^{2}\right)^{1 / 2}\right] d t d x
\end{aligned}
$$

The second integral in (5) can be integrated once by parts to yield

$$
\begin{aligned}
\mathcal{E}_{3}(\tau, \beta)= & \tau \int_{1}^{\infty} \exp \left[-\tau\left(t^{2}+\beta^{2}\right)^{1 / 2}\right] d t-\tau \mathcal{E}_{2}(\tau, \beta) \\
& -\tau \int_{1}^{\infty} \int_{1}^{\infty} x^{-2} \exp \left[-\tau\left(t^{2} x^{2}+\beta^{2}\right)^{1 / 2}\right] d t d x
\end{aligned}
$$

Since the double integral in (6) is another form of $\varepsilon_{3}(\tau, \beta),(6)$ can be rewritten as

$$
2 \varepsilon_{3}(\tau, \beta)=\tau \int_{1}^{\infty} \exp \left[-\tau\left(t^{2}+\beta^{2}\right)^{1 / 2}\right] d t-\tau \varepsilon_{2}(\tau, \beta)
$$

When $\beta=0$, (7) reduces to the standard one-dimensional form

$$
2 E_{3}(\tau)=\exp (-\tau)-\tau E_{2}(\tau)
$$

An expression for $\varepsilon_{3}(\tau, \beta)$ which depends only upon $\varepsilon_{1}(\tau, \beta)$ and $\varepsilon_{2}(\tau, \beta)$ can be obtained by eliminating the integral term in (7). Differentiating $\varepsilon_{1}(\tau, \beta)$ and substituting the result into (7), we get

$$
2 \varepsilon_{3}(\tau, \beta)=-\tau d \varepsilon_{1}(\tau, \beta) / d \tau-\tau \varepsilon_{2}(\tau, \beta) .
$$

This equation, along with (3), enables $\varepsilon_{3}(\tau, \beta)$ to be expressed in terms of either $\varepsilon_{1}(\tau, \beta)$ or $\varepsilon_{2}(\tau, \beta)$.

3. Series Expansions. Since neither $\mathcal{E}_{1}(\tau, \beta)$ nor $\mathcal{E}_{2}(\tau, \beta)$ can be integrated in closed form, series representations are necessary. A Taylor series expansion of $\varepsilon_{2}(\tau, \beta)$ about $\beta=0$ yields

$$
\begin{aligned}
E_{2}(\tau, \beta)= & E_{2}(\tau)-\frac{\tau}{2} E_{3}(\tau) \beta^{2}+\frac{\tau}{8}\left[\tau E_{4}(\tau)+E_{5}(\tau)\right] \beta^{4} \\
& -\frac{\tau}{48}\left[\tau^{2} E_{5}(\tau)+3 \tau E_{8}(\tau)+3 E_{7}(\tau)\right] \beta^{6} \\
& +\frac{\tau}{384}\left[\tau^{3} E_{8}(\tau)+6 \tau^{2} E_{7}(\tau)+15 \tau E_{8}(\tau)+15 E_{9}(\tau)\right] \beta^{8} \\
(10) \quad & -\frac{\tau}{3840}\left[\tau^{4} E_{7}(\tau)+10 \tau^{3} E_{8}(\tau)+45 \tau^{2} E_{9}(\tau)+105 \tau E_{10}(\tau)\right. \\
& +\frac{\tau}{46080}\left[\tau^{5} E_{8}(\tau)+15 \tau^{4} E_{9}(\tau)+105 \tau^{3} E_{10}(\tau)+105 \tau^{2} E_{11}(\tau)\right. \\
& \left.+945 \tau E_{12}(\tau)+945 E_{13}(\tau)\right] \beta^{12}-\ldots .
\end{aligned}
$$


A series representation for large values of $\beta$ is best obtained from expression (1a) for $\varepsilon_{1}(\tau, \beta)$. The substitution $t=\beta \sinh \xi$ reduces $\varepsilon_{1}(\tau, \beta)$ to a form so that an integral listed in [5] can be used. This integral

$$
K_{0}(\tau \beta)=\int_{0}^{\infty} \exp (-\tau \beta \cosh \xi) d \xi
$$

reduces (1a) to the form

$$
\varepsilon_{1}(\tau, \beta)=K_{0}(\tau \beta)-\int_{0}^{c} \exp (-\tau \beta \cosh \xi) d \xi
$$

where $c=\sinh ^{-1}(1 / \beta)$. The integrand in (12) is expanded in a series around $\xi=0$ and integrated to yield

$$
\begin{aligned}
& \mathcal{E}_{1}(\tau, \beta)=K_{0}(x) \\
& \begin{aligned}
&-c e^{-x}[ 1-\frac{x c^{2}}{3 !}+\frac{x(3 x-1) c^{4}}{5 !} \\
&-\frac{x\left(15 x^{2}-15 x+1\right) c^{6}}{7 !}+\frac{x\left(105 x^{3}-210 x^{2}+63 x-1\right) c^{8}}{9 !} \\
&-\frac{x\left(945 x^{4}-3150 x^{3}+2205 x^{2}-255 x+1\right) c^{10}}{11 !} \\
&+\frac{x\left(10395 x^{5}-51975 x^{4}+65835 x^{3}-21120 x^{2}+1023 x-1\right) c^{12}}{13 !} \\
&-x\left(135135 x^{6}-945945 x^{5}+1891890 x^{4}-1201200 x^{3}\right. \\
&\text { (13) } \left.\left.\quad+195195 x^{2}-4095 x+1\right) c^{14} / 15 !+\cdots\right]
\end{aligned}
\end{aligned}
$$

where $x=\tau \beta$.

Continual integration by parts of integral (1a) yields the following asymptotic expansion of $\mathcal{E}_{1}(\tau, \beta)$ for large $\tau$ :

$\varepsilon_{1}(\tau, \beta) \sim \exp \left[-\tau\left(1+\beta^{2}\right)^{1 / 2}\right]\left[\frac{1}{\tau}-\frac{\left(1+\beta^{2}\right)^{1 / 2}}{\tau^{2}}+\frac{\left(2+3 \beta^{2}\right)}{\tau^{3}}\right.$

$$
\begin{array}{r}
-\frac{\left(1+\beta^{2}\right)^{1 / 2}\left(6+15 \beta^{2}\right)}{\tau^{4}}+\frac{24+120 \beta^{2}+105 \beta^{4}}{\tau^{6}} \\
\left.-\frac{\left(1+\beta^{2}\right)^{1 / 2}\left(120+840 \beta^{2}+945 \beta^{4}\right)}{\tau^{6}}+\cdots\right] .
\end{array}
$$

This expansion reduces to the asymptotic expansion [6] for the exponential integral when $\beta=0$. Neglecting higher order terms in (14) the generalized exponential integral can be approximated by

$$
\mathcal{E}_{1}(\tau, \beta) \sim \exp \left[-\tau\left(1+\beta^{2}\right)^{1 / 2}\right] /\left[\tau+\left(1+\beta^{2}\right)^{1 / 2}\right] .
$$

4. Associated Functions. The generalized exponential integral functions can be expressed in terms of other functions. In particular, the generalized integral $E(a, x)$ is defined [7] as 


$$
E(a, x)=\int_{0}^{x}\left(t^{2}+a^{2}\right)^{-1 / 2}\left\{1-\exp \left[-\left(t^{2}+a^{2}\right)^{1 / 2}\right]\right\} d t
$$

and is related to $\varepsilon_{1}(\tau, \beta)$ as follows:

$$
\mathcal{E}_{1}(\tau, \beta)=K_{0}(\tau \beta)-\sinh ^{-1}(1 / \beta)+E(\tau \beta, \tau) .
$$

The function $E(a, x)$ is tabulated in [7] using numerical integration. Another special function, the incomplete modified Bessel function $K_{0}(w, z)$, is defined [8] as

$$
K_{0}(w, z)=\int_{0}^{w} \exp (-z \cosh t) d t
$$

and is related to $\varepsilon_{1}(\tau, \beta)$ as follows:

$$
\mathcal{E}_{1}(\tau, \beta)=K_{0}(\tau \beta)-K_{0}\left(\sinh ^{-1}(1 / \beta), \tau \beta\right) .
$$

In [7], [8], an asymptotic expansion equivalent to the first term of Eq. (14) is presented. However, no series expansions suitable for numerical computation are developed.

In studying the absorption of solar radiation by the earth's atmosphere, Chapman [9] studied the following function:

$$
f(x, \sin \sigma)=x \sin \sigma e^{x} \int_{U}^{\infty} \exp [-x \sin \sigma \cosh t] \cosh t d t
$$

where sech $U=\sin \sigma$. Chapman's function is related to the derivative of $\mathcal{E}_{1}(\tau, \beta)$, i.e., (21) . $-\tau d \varepsilon_{1}(\tau, \beta) / d \tau=\exp \left[-\tau\left(1+\beta^{2}\right)^{1 / 2}\right] f\left[\tau\left(1+\beta^{2}\right)^{1 / 2}, \beta\left(1+\beta^{2}\right)^{-1 / 2}\right]$.

5. Numerical Computation. Numerical values of $\mathcal{E}_{1}, \mathcal{E}_{2}$ and $\varepsilon_{3}$ are listed in Tables 1,2 and 3 in the microfiche section. The calculations were performed on an IBM 360 model 50 computer with double precision arithmetic. For $\beta \leqq .5$ and $\tau \leqq 2$, Eq. (10) was used to compute $\varepsilon_{2}(\tau, \beta)$ with $\varepsilon_{1}(\tau, \beta)$ and $\varepsilon_{3}(\tau, \beta)$ following from recursion relations (3) and (9). For $\beta>.5$ and $\tau \leqq 2$, Eq. (13) was used to compute $\mathcal{E}_{1}(\tau, \beta)$ with $\mathcal{E}_{2}(\tau, \beta)$ and $\mathcal{E}_{3}(\tau, \beta)$ following from recursion relations (3) and (9). For $\tau>2$, the generalized exponential integrals $\mathcal{E}_{n}(\tau, \beta)$ were calculated using Gaussian quadrature.

Using results calculated with Gaussian quadrature as a reference, the results from Eq. (10) yield six significant digits in the region $\beta \leqq .5$ and $\tau \leqq 2$. Results from Eq. (13) and those from the numerical quadrature differ by two units in the fifth significant digit for $\beta=.5$ and $\tau \leqq 2$. The accuracy of Eq. (10) increases as $\tau$ or $\beta$ decreases, while the accuracy of Eq. (13) increases as $\tau$ or $1 / \beta$ decreases. For example, the results from Eq. (10) yield seven significant digits for $\beta \leqq .5$ and $\tau \leqq 1$. Also, the results for $\varepsilon_{1}(\tau, \beta)$ were spot-checked with those of [7] and found in agreement.

Thermal Radiative Transfer Group

Department of Mechanical and Aerospace Engineering University of Missouri-Rolla

Rolla, Missouri 65401

1. M. G. SmITH, "The transport equation with plane symmetry and isotropic scattering," Proc. Cambridge Philos. Soc., v. 60, 1964, pp. 909-220. MR 32 \#327.

2. G. E. HuNT, "The transport equation of radiative transfer with axial symmetry," SIAM J. Appl. Math., v. 16, 1968, pp. 228-237. MR 37 \#143. 
3. G. B. RYBICKI, "The searchlight problem with isotropic scattering," J. Quant. Spectrosc. Radiat. Transfer, v. 11, 1971, pp. 827-849.

4. W. F. BREIG \& A. L. CROSBIE, "Two-dimensional radiative equilibrium," J. Math. Anal. Appl. (To appear.)

5. Y. L. LUKE, Integrals of Bessel Functions, McGraw-Hill, New York, 1962, p. 30. MR 25 \#5198.

6. M. Abramowitz \& I. A. Stegun (Editors), Handbook of Mathematical Functions, With Formulas, Graphs and Mathematical Tables, Nat. Bur. Standards Appl. Math. Series, 55, Superintendent of Documents, U.S. Government Printing Office, Washington, D.C., 1964. MR 29 \#4914.

7. Tables of the Generalized Exponential Integral Functions, Harvard University, Annals of the Computation Laboratory. Vol. 21, Harvard Univ. Press, Cambridge, Mass., 1949. 8. M. M. Agrest \& M. S. Maksimov, Theory of Incomplete Cylindrical Functions and Their Applications, Springer-Verlag, Berlin and New York, 1971.

9. S. CHAPMAN, "The absorption and dissociative or ionizing effect of monochromatic radiation in an atmosphere on a rotating earth," Proc. Phys. Soc., v. 43, 1931, pp. 483-501. 